\title{
Norm-dependent Random Matrix Ensembles in External Field and Supersymmetry
}

\author{
Thomas Guhr \\ Matematisk Fysik, LTH, Lunds Universitet, Box 118, 22100 Lund, Sweden
}

\begin{abstract}
The class of norm-dependent Random Matrix Ensembles is studied in the presence of an external field. The probability density in those ensembles depends on the trace of the squared random matrices, but is otherwise arbitrary. An exact mapping to superspace is performed. A transformation formula is derived which gives the probability density in superspace as a single integral over the probability density in ordinary space. This is done for orthogonal, unitary and symplectic symmetry. In the case of unitary symmetry, some explicit results for the correlation functions are derived.

PACS numbers: 05.45.Mt, 05.30.-d, 02.30.Px
\end{abstract}

\section{Introduction}

Supersymmetry is a prominent and widely used tool in studying disordered systems and systems that can be modeled by random matrices, see Refs. [1, 2, 3, 4, 5, 6]. The method was developed for Gaussian probability densities, a review and a discussion of the mathematical justification was recently given in Ref. [7]. This restriction to Gaussian probability densities is no shortcoming if one is exclusively interested in calculating correlations on the local scale of the mean level spacing. This is due to local universality [8, 9]. Probability densities which do not introduce scales competing with the mean level spacing yield correlations which are one the local scale identical to the ones resulting from Gaussian probability densities, see a review in Ref. [4. When studying matrix models in high-energy physics one is not interested in the local scale. Another universality in the leading asymptotics of the matrix dimension was found [10] for the correlation functions on large scales.

Nevertheless, restriction to Gaussian probability densities does not always suffice. First, the one-point functions obviously depend on the specific form of the probability densities, because they are not measured on local scales. Such level densities are important, for example for applications in high energy physics [11], but also in finance [12]. Second, to distinguish certain directions in matrix space, one adds an external field to the random matrix, and one often averages over the matrices representing the external field. The local correlations now change and depend sensitively on the root-mean square matrix element of the external field divided by the local mean 
level spacing. Examples are the crossover transitions, see the review in Refs. [4, 5]. In such a situation, scales competing with the local mean level spacing might occur which can lead to a deviation from universal features, such that the crossover transitions would differ for different probability densities. Third, non-Gaussian probability densities and their non-universal features on special scales have always been of interest in conceptual studies and for considerations in general statistical mechanics, we mention the boundtrace and the fixed-trace ensembles [13] and the recently introduced ensembles deriving from a non-extensive entropy principle [14, 15].

In the present contribution, we show that the supersymmetry method can be extended to random matrix models with non-Gaussian probability densities. In the context of universality, asymptotic considerations for infinite level number have already been combined with supersymmetric techniques for non-Gaussian probability densities in Ref. [16]. Here, however, we aim at an exact discussion. We focus on the large class of norm-dependent random matrix ensembles which depend through an arbitrary functional form on the trace of the squared random matrices. Recently, a general construction of these ensembles was given in Ref. [17]. For the reasons just mentioned, we include an external field. We have two goals. First, we want to deliver the conceptually important insight that supersymmetry is by no means restricted to Gaussian probability densities. Second, we want to provide a series of explicit and practically relevant formulae for the correlation functions. Here, the application of supersymmetry yields particularly handy results in the presence of an external field.

The question whether or not norm-dependent ensembles can be formulated exactly in a supersymmetric framework was also discussed by F. Kalisch. Although his approach was quite different from the one to be presented here, it would have been likely to produce equivalent results for the case without external field. Unfortunately, F. Kalisch left academia and his findings are unpublished.

The paper is organized as follows. We formulate the problem in Section 2 , thereby also introducing our notations and conventions. The supersymmetric representation of the norm-dependent ensembles is constructed in Section 3, In Section 4, we discuss a series of examples. Explicit results for the correlation functions are given in Section 5 . We summarize and conclude in Section 6.

\section{Formulation of the Problem}

In Section 2.1, we set up the generating function in the presence of an external field. As we need to refer to the Gaussian case, we briefly sketch it in Section 2.2. We discuss norm-dependent ensembles and pose the problem in Section 2.3.

\subsection{Generating Function in the Presence of an External Field}

The three symmetry classes of $N \times N$ random matrices $H$ are labeled by the Dyson index $\beta$. In the orthogonal class, $H$ is real symmetric $(\beta=1)$ and in the unitary class, $H$ 
is Hermitean $(\beta=2)$. In the symplectic class, $H$ is self-dual $(\beta=4)$ and the entries of $H$ are $2 \times 2$ quaternions. The eigenvalues of $H$ are doubly degenerate in the symplectic class. We notice that such a matrix has

$$
\mu=N+\frac{\beta}{2} N(N-1)
$$

independent matrix elements. The quantity $\mu$ is often referred to as the number of degrees of freedom. A normalized probability density function $P^{(\beta)}(H)$ defines together with the symmetry class - the random matrix ensemble. We add a fixed external field represented by a matrix $H_{0}$ which, without loss of generality, can be assumed to be diagonal. In the symplectic class, it has dimension $2 N \times 2 N$. Thus, we are interested in a system described by $H_{0}+\alpha H$ where $\alpha$ measures the relative strength. The $k$ level correlation function $R_{k}^{(\beta)}\left(x_{1}, \ldots, x_{k}, \alpha, H_{0}\right)$ is the probability density to find $k$ eigenvalues of $H_{0}+\alpha H$ at positions $x_{1}, \ldots, x_{k}$. The correlation functions $\widehat{R}_{k}^{(\beta)}\left(x_{1}, \ldots, x_{k}, \alpha, H_{0}\right)$ are technically easier to handle. They include real and imaginary parts of the propagator, while the $R_{k}^{(\beta)}\left(x_{1}, \ldots, x_{k}, \alpha, H_{0}\right)$ are only the correlations of the imaginary parts. The latter can easily be constructed from the former. We use the conventions of Refs. [18, 19, 20]. For arbitrary $P^{(\beta)}(H)$, the correlation function can be written as the derivative

$$
\widehat{R}_{k}^{(\beta)}\left(x_{1}, \ldots, x_{k}, \alpha, H_{0}\right)=\left.\frac{1}{(2 \pi)^{k}} \frac{\partial^{k}}{\prod_{p=1}^{k} \partial J_{p}} Z_{k}^{(\beta)}(x+J)\right|_{J_{p}=0}
$$

of a generating function

$$
Z_{k}^{(\beta)}(x+J)=\int d[H] P^{(\beta)}(H) \prod_{p=1}^{k}\left(\frac{\operatorname{det}\left(H_{0}+\alpha H-x_{p}-J_{p}\right)}{\operatorname{det}\left(H_{0}+\alpha H-x_{p}+J_{p}\right)}\right)^{\gamma}
$$

with respect to source variables $J_{p}, p=1, \ldots, k$. Here, we define $\gamma=1$ if $\beta=1,2$ and $\gamma=2$ if $\beta=4$, moreover we introduce the diagonal matrices $x=\operatorname{diag}\left(x_{1}, x_{1}, \ldots, x_{k}, x_{k}\right)$ and $x=\operatorname{diag}\left(-J_{1},+J_{1}, \ldots,-J_{k},+J_{k}\right)$. The volume element $d[H]$ is simply the product of the differentials of all independent matrix elements. For complex variables, we use the differentials of real and imaginary part.

\subsection{Gaussian Random Matrix Ensembles}

In the Gaussian case, the normalized probability density function with variance $2 v^{2} / \beta$ reads

$$
P^{(G \beta)}(H)=\frac{1}{2^{N / 2}}\left(\frac{\beta}{2 \pi v^{2}}\right)^{\mu / 2} \exp \left(-\frac{\beta}{4 v^{2}} \operatorname{Tr} H^{2}\right)
$$

with the number $\mu$ of degrees of freedom given in Eq. (11). To properly account for the degeneracies in the symplectic class, we define

$$
\operatorname{Tr}=\left\{\begin{array}{ll}
\operatorname{tr} & \text { if } \quad \beta=1,2 \\
\frac{1}{2} \operatorname{tr} & \text { if } \beta=4
\end{array} .\right.
$$


The probability density (44) and the symmetry class define the Gaussian Orthogonal, Unitary and Symplectic Ensemble GOE, GUE and GSE for $\beta=1,2,4$, respectively. The generating function

$$
\begin{aligned}
Z_{k}^{(G \beta)}\left(x+J, 2 v^{2} / \beta\right)=\int d[H] & P^{(G \beta)}(H) \\
& \prod_{p=1}^{k}\left(\frac{\operatorname{det}\left(H_{0}+\alpha H-x_{p}-J_{p}\right)}{\operatorname{det}\left(H_{0}+\alpha H-x_{p}+J_{p}\right)}\right)^{\gamma}
\end{aligned}
$$

of this case has an exact representation as integral in superspace,

$$
\begin{aligned}
Z_{k}^{(G \beta)}\left(x+J, 2 v^{2} / \beta\right)= & \int d[\sigma] Q^{(G \beta)}(\sigma) \\
& \operatorname{detg}^{-\beta / 2 \gamma}\left((\alpha \sigma-x-J) \otimes 1_{\gamma N}+1_{\zeta k} \otimes H_{0}\right) .
\end{aligned}
$$

The crucial feature of supersymmetry is the drastic reduction in the number of degrees of freedom. This is borne out in the dimension of the matrix $\sigma$. It is a $2 k \times 2 k$ Hermitean supermatrix for $\beta=2$ and a $4 k \times 4 k$ Hermitean supermatrix with additional symmetries for $\beta=1,4$ [1], we use the conventions of Refs. [21, 22]. The parameter $\zeta=2$ for $\beta=2$ and $\zeta=4$ for $\beta=1,4$ is defined accordingly. We write $1_{M}$ for the $M \times M$ unit matrix. Thus, the expression (17) contains the unit matrices $1_{N}$ and $1_{4 k}$ for $\beta=1,1_{N}$ and $1_{2 k}$ for $\beta=2$ and $1_{2 N}$ and $1_{4 k}$ for $\beta=4$. Again, the volume element $d[\sigma]$ is the product of the differentials of all independent variables. For the complex anticommuting variables, we use the differentials of the variable and of its complex conjugate. The probability density in superspace

$$
Q^{(G \beta)}(\sigma)=c^{(\beta)} \exp \left(-\frac{\beta}{4 v^{2}} \operatorname{trg} \sigma^{2}\right)
$$

is a normalized Gaussian as well. Importantly, the normalization constants

$$
c^{(\beta)}= \begin{cases}2^{k(k-1)} & \text { if } \quad \beta=2 \\ 2^{k(4 k-3) / 2} & \text { if } \quad \beta=1,4\end{cases}
$$

depend only on the dimension. In contrast to the ordinary case (41), they doe not contain the variance $2 v^{2} / \beta$.

The result (7) has a remarkable property. The superdeterminant comprises a sum of two terms which are both direct products. The first term is a direct product of supermatrices with a unit matrix and ordinary space, and vice versa in the second term. Most conveniently, this decouples to some extent the random matrix ensemble, i.e. the matrix $\sigma$ from the external field $H_{0}$. This feature, which is typical for the supersymmetry method, was already very helpful for an exact calculation of the transition from Poisson regularity to the GUE in Refs. [19, 20]. Furthermore, it also made possible some asymptotic evaluation [23, 24] of the correlations on the local scale for large coupling $\alpha / D$ where $D$ is the mean level spacing.

\subsection{Posing the Problem for Norm-dependent Ensembles}

In analogy to the scalar product for vectors, one introduces a scalar product $\operatorname{Tr} H K$ for two matrices $H$ and $K$ with the same symmetries. This is then used to define the norm 
of a matrix by

$$
\|H\|=\sqrt{\operatorname{Tr} H^{2}}
$$

corresponding to the length of a vector. The class of norm-dependent ensembles has a probability density of the form

$$
P^{(\beta)}(H)=P^{(T \beta)}\left(\operatorname{Tr} H^{2}\right),
$$

where $P^{(T \beta)}(u)$ is function of the norm $\|H\|$ or, equivalently of $u=\operatorname{Tr} H^{2}$. Of course, $P^{(T \beta)}(u)$ has to be chosen such that $P^{(\beta)}(H)$ is positive semi-definite and fulfills all the necessary convergence requirements. According to the symmetries, there are Normdependent Orthogonal, Unitary and Symplectic Ensembles for $\beta=1,2,4$. We denote them TOE, TUE and TSE, respectively. We show in Appendix A that the $\nu$-th moment of the probability density can be expressed, if it exists, as the single integral

$$
\begin{aligned}
M_{\nu}^{(T \beta)} & =\int P^{(T \beta)}\left(\operatorname{Tr} H^{2}\right)\left(\operatorname{Tr} H^{2}\right)^{\nu} d[H] \\
& =\left(\frac{\pi}{2}\right)^{\mu / 2} \frac{2^{N / 2}}{\Gamma(\mu / 2)} \int_{0}^{\infty} u^{\nu+\mu / 2-1} P^{(T \beta)}(u) d u .
\end{aligned}
$$

This includes the normalization by setting $M_{0}^{(T \beta)}=1$ for $\nu=0$.

Many ensembles fall into the norm-dependent class. Obviously, the Gaussian Ensembles are found by setting $P^{(T \beta)}(u) \sim \exp \left(-\beta u / 4 v^{2}\right)$, which is an exponential function, not a Gaussian. Non-trivial examples are the fixed-trace and the bound-trace ensembles [13. An important subclass of norm-dependent ensembles is derived from a non-extensive entropy principle [14, 15]. It comprises a variety of interesting cases which are found by considering limits of certain parameter. A rather general construction of norm-dependent ensembles using a single-valued spread function is given in Ref. [17. We return to this point.

We ask the following questions. Can we express the generating function for the norm-dependent ensembles TOE, TUE and TSE given by

$$
\begin{aligned}
Z_{k}^{(T \beta)}(x+J)=\int d[H] P^{(T \beta)} & \left(\operatorname{Tr} H^{2}\right) \\
& \prod_{p=1}^{k}\left(\frac{\operatorname{det}\left(H_{0}+\alpha H-x_{p}-J_{p}\right)}{\operatorname{det}\left(H_{0}+\alpha H-x_{p}+J_{p}\right)}\right)^{\gamma}
\end{aligned}
$$

as integral in superspace? — Can we construct the supersymmetric analog of the probability density $P^{(T \beta)}\left(\operatorname{Tr} H^{2}\right)$ ? - The answers are in the affirmative. We will derive the exact representation

$$
\begin{aligned}
Z_{k}^{(T \beta)}(x+J)=\int & d[\sigma] Q^{(T \beta)}\left(\operatorname{trg} \sigma^{2}\right) \\
& \operatorname{detg}^{-\beta / 2 \gamma}\left((\alpha \sigma-x-J) \otimes 1_{\gamma N}+1_{\zeta k} \otimes H_{0}\right),
\end{aligned}
$$

where the supermatrices $\sigma$ are defined as above and where the probability density $Q^{(T \beta)}\left(\operatorname{trg} \sigma^{2}\right)$ is also norm-dependent, but now in superspace.

Importantly, the direct product structure implying the decoupling of the random matrix ensemble from the external field $H_{0}$ is also present here for all TOE, TUE and TSE. This extends the discussion in Section 2.2 for the Gaussian ensembles. 


\section{Supersymmetric Representation}

In Section 3.1, we derive the supersymmetric representation by using Fourier integrals. We present the transformation formulae for the probability densities in Section 3.2 . The connection to the spread function is discussed in Section 3.3, which also contains an alternative derivation of the transformation formulae.

\subsection{Derivation Using Fourier Integrals}

The norm $\|H\|$ is non-negative and we have $u=\operatorname{Tr} H^{2} \geq 0$. Thus, $P^{(T \beta)}(u)$ is only defined on the positive $u$ axis. When introducing the Fourier integral over the entire axis, we have to set $P^{(T \beta)}(u)=0$ for $u<0$, such that

$$
p^{(T \beta)}(y)=\frac{1}{\sqrt{2 \pi}} \int_{0}^{\infty} P^{(T \beta)}(u) \exp (i y u) d u
$$

is the Fourier transform with the inversion

$$
P^{(T \beta)}(u)=\frac{1}{\sqrt{2 \pi}} \int_{-\infty}^{+\infty} p^{(T \beta)}(y) \exp (-i y u) d y .
$$

We add a small imaginary increment to the Fourier variable, $y^{-}=y-i \varepsilon$ and insert insert Eq. (16) with $u=\operatorname{Tr} H^{2}$, i.e. the integral

$$
P^{(T \beta)}\left(\operatorname{Tr} H^{2}\right)=\frac{1}{\sqrt{2 \pi}} \int_{-\infty}^{+\infty} p^{(T \beta)}(y) \exp \left(-i y^{-} \operatorname{Tr} H^{2}\right) d y .
$$

into the generating function (13). We thereby rediscover the Gaussian case (6) with the variance $1 / i 2 y^{-}$. The integrals over $H$ can now be done as Gaussian integrals, the complex variance $1 / i 2 y^{-}$does not cause a problem. Even without the imaginary increment, they exist as Fresnel integrals. The imaginary increment makes standard Gaussian integrals out of them, but this is not the motivation for it. We need the imaginary increment later on. The important difference to the Gaussian case of Section 2.2 is the fact that the Gaussian $\exp \left(-i y^{-} \operatorname{Tr} H^{2}\right)$ comes without normalization constant for the $H$ integration. Hence, when inserting Eq. (17) into Eq. (6) we obtain the inverse of the normalization constant as an $y$ dependent factor in the Fourier integral,

$$
\begin{aligned}
Z_{k}^{(T \beta)}(x+J)=\frac{1}{\sqrt{2 \pi}} \int_{-\infty}^{+\infty} d y p^{(T \beta)}(y) \\
2^{N / 2}\left(\frac{\pi}{i 2 y^{-}}\right)^{\mu / 2} Z_{k}^{(G \beta)}\left(x+J, 1 / i 2 y^{-}\right)
\end{aligned}
$$

with $Z_{k}^{(G \beta)}\left(x+J, 1 / i 2 y^{-}\right)$given in Eq. (6). We now employ the supersymmetric representation (7) and find

$$
Z_{k}^{(T \beta)}(x+J)=\frac{1}{\sqrt{2 \pi}} \int_{-\infty}^{+\infty} d y p^{(T \beta)}(y) 2^{N / 2}\left(\frac{\pi}{i 2 y^{-}}\right)^{\mu / 2}
$$




$$
\begin{aligned}
& \int d[\sigma] c^{(\beta)} \exp \left(-i y^{-} \operatorname{trg} \sigma^{2}\right) \\
& \quad \operatorname{detg}^{-\beta / 2 \gamma}\left((\alpha \sigma-x-J) \otimes 1_{\gamma N}+1_{\zeta k} H_{0}\right) .
\end{aligned}
$$

Hence, by interchanging the integrations, we arrive at the desired Eq. (14), where the probability density in superspace

$$
Q^{(T \beta)}\left(\operatorname{trg} \sigma^{2}\right)=c^{(\beta)} \frac{2^{N / 2}}{\sqrt{2 \pi}}\left(\frac{\pi}{2}\right)^{\mu / 2} \int_{-\infty}^{+\infty} p^{(T \beta)}(y) \frac{\exp \left(-i y^{-} \operatorname{trg} \sigma^{2}\right)}{\left(i y^{-}\right)^{\mu / 2}} d y .
$$

is the inverse Fourier integral with an additional power $\left(i y^{-}\right)^{\mu / 2}$ in the denominator.

\subsection{Transformation Formulae}

We set $w=\operatorname{trg} \sigma^{2}$ and plug the Fourier integral (15) into Eq. (20),

$$
Q^{(T \beta)}(w)=c^{(\beta)} \frac{2^{N / 2}}{2 \pi}\left(\frac{\pi}{2}\right)^{\mu / 2} \int_{0}^{\infty} d u P^{(T \beta)}(u) \int_{-\infty}^{+\infty} \frac{\exp (-i y(u-w))}{\left(i y^{-}\right)^{\mu / 2}} d y .
$$

The $y$ integral converges because of the imaginary increment and can be done in a standard way 33. Apart from factors, it yields $\Theta(u-w)(u-w)^{\mu / 2-1}$. We thus arrive at the transformation formula

$$
Q^{(T \beta)}(w)=\frac{c^{(\beta)} 2^{N / 2}}{\Gamma(\mu / 2)}\left(\frac{\pi}{2}\right)^{\mu / 2} \int_{0}^{\infty} P^{(T \beta)}(u+w) u^{\mu / 2-1} d u .
$$

This result allows one to calculate, by a single integration, the probability density in superspace for any norm-dependent ensemble TOE, TUE and TSE. We notice that the Fourier integral (15) has to exist, but, importantly, its explicit knowledge is not needed to obtain the probability density $Q^{(T \beta)}(w)$ in superspace. Interestingly, the transformation formula can be inverted. For even number of degrees of freedom $\mu$, iterated integration by parts yields

$$
P^{(T \beta)}(u)=\frac{(-1)^{\mu / 2}}{c^{(\beta)} 2^{N / 2}}\left(\frac{2}{\pi}\right)^{\mu / 2} \frac{\partial^{\mu / 2}}{\partial u^{\mu / 2}} Q^{(T \beta)}(u) .
$$

This inversion is likely to be correct even for odd $\mu$ if the theory of fractional derivatives is applied.

From a conceptual viewpoint, the pair of transformation formulae (22) and (23) states the main result of this contribution. The power of supersymmetry lies in the drastic reduction of the degrees of freedom. The mechanism of how this happens was previously only known in the Gaussian case. The transformation formulae (22) and (23) considerably generalize that. A particularly interesting interpretation follows from formula (23). The probability densities $P^{(T \beta)}(u)$ and $Q^{(T \beta)}(u)$ formally coincide for $\mu=0$, i.e. in zero dimensions, $N=0$. This is already visible in the Gaussian case. Apart from the variance independent normalization $c^{(\beta)}$, the Gaussian (8) in superspace with $u=\operatorname{trg} \sigma^{2}$ indeed follows from the Gaussian (4) in ordinary space with $u=\operatorname{Tr} H^{2}$ by simply setting $N=0$. 
Another interesting observation results from putting $w=0$ in the transformation formula (22) and then using the normalization of $P^{(T \beta)}(u)$ which can be read off from Eq. (12) for $\nu=0$. One has $Q^{(T \beta)}(0)=c^{(\beta)}$. In other words, the normalization of the probability density in ordinary space corresponds to the value of the probability density in superspace at $w=0$. This reflects the Efetov-Wegner-Parisi-Sourlas theorem [1, 25, 26, 27], referred to as Rothstein theorem in mathematics [28]. It implies that the normalization integral for a function such as ours which only depends on invariants reads

$$
1=\int Q^{(T \beta)}\left(\operatorname{trg} \sigma^{2}\right) d[\sigma]=\frac{1}{c^{(\beta)}} Q^{(T \beta)}(0) .
$$

This phenomenon exclusively occurs in superspace is due to a subtle mutual cancellation of singularities. Hence, it is reassuring to see that the normalization of the probability density in ordinary space leads — via the Efetov-Wegner-Parisi-Sourlas theorem - to the normalization of the probability density in superspace.

\subsection{Connection to the Spread Function}

A rather general construction of norm-dependent ensembles was given by Muttalib and Klauder [17] for the unitary case. It can be generalized to all three symmetry classes in a straightforward manner. The probability density

$$
P^{(T \beta)}\left(\operatorname{Tr} H^{2}\right)=\int_{0}^{\infty} f^{(T \beta)}(t) \frac{1}{2^{N / 2}}\left(\frac{\beta}{2 \pi t}\right)^{\mu / 2} \exp \left(-\frac{\beta}{4 t} \operatorname{Tr} H^{2}\right) d t
$$

is expressed as an integral involving a normalized Gaussian with a real variance $2 t / \beta$. The quantity $f^{(T \beta)}(t)$ is referred to as spread function. As seen form Eq. (25) it is normalized,

$$
\int_{0}^{\infty} f^{(T \beta)}(t) d t=1
$$

We insert the integral (25) into the generating function (13) and find in steps analogous to the ones in Section 3.1 .

$$
\begin{aligned}
& Z_{k}^{(T \beta)}(x+J)=\int_{0}^{\infty} d t f^{(T \beta)}(t) Z_{k}^{(G \beta)}(x+J, 2 t / \beta) \\
&=\int_{0}^{\infty} d t f^{(T \beta)}(t) \int d[\sigma] c^{(\beta)} \exp \left(-\frac{\beta}{4 t} \operatorname{trg} \sigma^{2}\right) \\
& \operatorname{detg}^{-\beta / 2 \gamma}\left((\alpha \sigma-x-J) \otimes 1_{\gamma N}+1_{\zeta k} H_{0}\right) .
\end{aligned}
$$

This yields again Eq. (14) where the probability density in superspace now reads

$$
Q^{(T \beta)}\left(\operatorname{trg} \sigma^{2}\right)=\int_{0}^{\infty} f^{(T \beta)}(t) c^{(\beta)} \exp \left(-\frac{\beta}{4 t} \operatorname{trg} \sigma^{2}\right) d t .
$$

Comparing Eqs. (25) and (28) one sees that the probability densities are in ordinary and in superspace given as integrals over the spread function times a normalized Gaussian. 
Moreover, we notice that the variable $t$ in the variance $2 t / \beta$ has the meaning of a diffusion time. In ordinary space, the diffusion is Dyson's Brownian Motion [29, 30]. It has a fully fledged analog in superspace [20] with the same diffusion time. Thus, the TOE, TUE and TSE are, in ordinary and in superspace, ensembles constructed as averages involving the diffusion time.

The transformation formulae (22) and (23) are easily re-derived from Eqs. (25) and (28). We emphasize that only the existence, but not the precise knowledge of the spread function is needed to calculate the probability density in superspace. Those readers might appreciate the alternative derivation of the transformation formulae by means of the spread function who did not feel comfortable with our treatment of the singularities in the Fourier integrals of Section 3.1

\section{Some Specific Examples}

To gain insight into how the transformation formulae work, we calculate the probability densities in superspace for a variety of examples. To acquire some first experience, we revisit the Gaussian ensembles in Section 4.1. We discuss, for all three symmetry classes $\beta=1,2,4$ the bound trace, the fixed trace, the Gauss-monomial and the Gaussquartic ensembles in Sections 4.2 to 4.5. For the probability densities in ordinary space of these examples, we introduce constants $a_{0}, a_{1}$ and $a_{2}$ which are always assumed to be real and positive. Using Eq. (12), they can be expressed in terms of the moments $M_{\nu}^{(T \beta)}$. In particular, the overall normalization constant can be fixed with Eq. (12)

for $\nu=0$. However, we rather use the relation $Q^{(T \beta)}(0)=c^{(\beta)}$ which is according to Section 3.2 equivalent to the normalization of the probability density in ordinary space. In Section 4.6 we discuss the ensembles derived from an non-extensive entropy principle. We always write $u=\operatorname{Tr} H^{2}$ and $w=\operatorname{trg} \sigma^{2}$.

\subsection{Revisiting the Gaussian Ensembles}

Inserting the Gaussian (44) into the transformation formula (22), we find

$$
\begin{array}{r}
Q^{(G \beta)}(w)=\exp \left(-\frac{\beta}{4 v^{2}} w\right) \frac{c^{(\beta)}}{\Gamma(\mu / 2)}\left(\frac{\beta}{4 v^{2}}\right)^{\mu / 2} \\
\int_{0}^{\infty} \exp \left(-\frac{\beta}{4 v^{2}} u\right) u^{\mu / 2-1} d u,
\end{array}
$$

which gives the Gaussian (8). The Fourier transform

$$
p^{(G \beta)}(y)=\frac{1}{\sqrt{2 \pi} 2^{N / 2}}\left(\frac{\beta}{2 \pi v^{2}}\right)^{\mu / 2} \frac{1}{i y-\beta / 4 v^{2}}
$$

can be also be used to infer the spread function, which is a $\delta$ function. The integral (25) has to be interpreted as a proper Cauchy integral. 


\subsection{Bound Trace Ensembles}

The probability density sets a cutoff for the norm of the random matrices according to 13

$$
P^{(B T \beta)}(u)=a_{0} \Theta\left(a_{1}-u\right) .
$$

The transformation formula (22) yields

$$
Q^{(B T \beta)}(w)=c^{(\beta)} \frac{\left(a_{1}-w\right)^{\mu / 2}}{a_{1}^{\mu / 2}} \Theta\left(a_{1}-w\right),
$$

which is a bound trace ensemble as well, but now in superspace and multiplied with a polynomial factor.

\subsection{Fixed Trace Ensembles}

The probability density fixes the norm of the random matrices such that [13, 31, 32,

$$
P^{(F T \beta)}(u)=a_{0} \delta\left(a_{1}-u\right) .
$$

With the transformation formula (22) we find

$$
Q^{(F T \beta)}(w)=c^{(\beta)} \frac{\left(a_{1}-w\right)^{\mu / 2-1}}{a_{1}^{\mu / 2-1}} \Theta\left(a_{1}-w\right),
$$

which is, once more, a bound trace ensemble of the form (32). We notice that the exponent in the polynomial factor is $\mu / 2-1$ compared with $\mu / 2$ in Eq. (32). This simply reflects that the probability density (33) is the derivative of the probability density (31). We mention that fixed trace ensembles do not seem to exist in superspace, at least not in a simple--minded interpretation. This is so, because the normalization requirement $Q^{(T \beta)}(0)=c^{(\beta)}$ can hardly be fulfilled if $Q^{(T \beta)}(w)$ includes a $\delta$ function.

\subsection{Gauss-Monomial Ensembles}

The probability densities in superspace derived in the previous examples tend to have remarkable similarity to the ones in ordinary space. This seems to be a fairly robust phenomenon. To illustrate it further, we introduce ensembles comprising a Gaussian and a monomial factor,

$$
P^{(G M \beta)}(u)=a_{0} u^{m} \exp \left(-a_{1} u\right),
$$

where $m$ is an integer. The transformation formula (22) implies

$$
Q^{(G M \beta)}(w)=c^{(\beta)} \exp \left(-a_{1} w\right) \sum_{m^{\prime}=0}^{m}\left(\begin{array}{c}
m \\
m^{\prime}
\end{array}\right) \frac{\Gamma\left(m-m^{\prime}+\mu / 2\right)}{\Gamma(m+\mu / 2)}\left(a_{1} w\right)^{m^{\prime}} .
$$

These are Gauss-polynomial ensembles including all powers between zero and $m$. 


\subsection{Gauss-Quartic Ensembles}

We now consider Gaussian probability densities supplemented with a quartic term in the exponent,

$$
P^{(G Q \beta)}(u)=a_{0} \exp \left(-a_{1} u-a_{2} u^{2}\right) .
$$

With the transformation formula (22), we obtain

$$
Q^{(G Q \beta)}(w)=c^{(\beta)} \exp \left(-\frac{a_{1}}{2} w-\frac{a_{2}}{2} w^{2}\right) \frac{D_{-\mu / 2}\left(a_{1} / \sqrt{2 a_{2}}+\sqrt{2 a_{2}} w\right)}{D_{-\mu / 2}\left(a_{1} / \sqrt{2 a_{2}}\right)},
$$

where $D_{p}(z)$ denotes the parabolic cylinder function of order $p$ [33]. Once more, the probability density in superspace contains the functional form of the one in ordinary space. However, this example also shows that one can come up with cases in which the additional contribution has a rather inconvenient structure.

\subsection{Ensembles Deriving from a Non-extensive Entropy Principle}

An interesting family of ensembles was constructed in Refs. [14, 15]. Among other features, it yields in a certain parameter range an invariant Lévy-type-of ensemble. The construction of Refs. [14, 15] is done for the orthogonal symmetry class, but can easily be generalized to all $\beta=1,2,4$. The probability density in ordinary space

$$
P^{(N E \beta)}(u)=a_{0}\left(1+\frac{\kappa}{\Lambda} u\right)^{1 /(1-q)}
$$

depends on a parameter $q$ used in the non-extensive entropy. Moreover, it includes a positive parameter $\kappa$ and

$$
\Lambda=\frac{1}{q-1}-\frac{\mu}{2}
$$

with $\mu$ being the number of degrees of freedom (11). To avoid confusion with the notation in the present contribution, we write $\kappa, \Lambda, \mu$, instead of $\alpha, \lambda, f$ in Ref. [15].

We consider $q>1$. This choice makes the exponent in the probability density (39) negative. Moreover, it requires $\Lambda>0$ such that

$$
1<q<q_{\max }=1+\frac{2}{\mu} .
$$

With help of the integral representation [15]

$$
P^{(N E \beta)}(u)=\frac{a_{0}}{\Gamma(1 /(q-1))} \int_{0}^{\infty} \xi^{1 /(q-1)-1} \exp \left(-\left(1+\frac{\kappa}{\Lambda} u\right) \xi\right) d \xi,
$$

we can obtain the probability density in superspace from the transformation formula (22). The $u$ integral has to be done first. Convergence is ensured because of the condition $\Lambda>0$. Collecting everything, we arrive at

$$
Q^{(N E \beta)}(w)=c^{(\beta)}\left(1+\frac{\kappa}{\Lambda} w\right)^{-\Lambda}
$$

Remarkably, this is again the same functional form as in ordinary space. The (negative) exponent $1 /(1-q)$ in ordinary space is mapped onto $-\Lambda$ in superspace. We notice that $Q^{(N E \beta)}(w)$ depends on $q$ only via the parameter $\Lambda$ which appears twice in Eq. (43). 


\section{Correlation Functions}

After discussing general results for all symmetry classes in Section 5.11, we give more explicit formulae for the unitary case in Section 5.2. All results to be given here can also be averaged over the external field $H_{0}$ with some probability density $P_{0}\left(H_{0}\right)$. However, as this is an obvious step, we do not go into that further.

\subsection{All Symmetry Classes}

We now have the supersymmetric representation (14) for the generating function and the one-dimensional transformation formula (22) for the probability density $Q^{(T \beta)}(w)$ in superspace. Hence, we can exploit the advantages of supersymmetry also for normdependent ensembles. In particular, the level number $N$ is, in contrast to the ordinary space, an explicit parameter in Eq. (14). Depending on the ensemble, this can makes it possible to study the generating function by means of a saddle point approximation for large $N$. As the details of such a calculation will sensitively depend on the specific form of $Q^{(T \beta)}(w)$, we refrain from attempting a general discussion.

We can also proceed by observing that Eqs. (18) and (27) are integral transforms of the generating functions involving the Fourier integral,

$$
Z_{k}^{(T \beta)}(x+J)=\frac{2^{N / 2} \pi^{\mu / 2}}{\sqrt{2 \pi}} \int_{-\infty}^{+\infty} \frac{p^{(T \beta)}(y)}{\left(i 2 y^{-}\right)^{\mu / 2}} Z_{k}^{(G \beta)}\left(x+J, 1 / i 2 y^{-}\right) d y,
$$

or the spread function,

$$
Z_{k}^{(T \beta)}(x+J)=\int_{0}^{\infty} f^{(T \beta)}(t) Z_{k}^{(G \beta)}(x+J, 2 t / \beta) d t
$$

respectively. Thus, the correlation functions $R_{k}^{(T \beta)}\left(x_{1}, \ldots, x_{k}, \alpha, H_{0}\right)$ of all normdependent ensembles are obtained as single integrals over the corresponding ones $R_{k}^{(G \beta)}\left(x_{1}, \ldots, x_{k}, \alpha, H_{0}\right)$ in the Gaussian case. With Eqs. (22) and (44), we find

$$
\begin{aligned}
& R_{k}^{(T \beta)}\left(x_{1}, \ldots, x_{k}, \alpha, H_{0}\right) \\
& =\frac{2^{N / 2} \pi^{\mu / 2}}{\sqrt{2 \pi}} \int_{-\infty}^{+\infty} d y \frac{p^{(T \beta)}(y)}{\left(i 2 y^{-}\right)^{\mu / 2}}\left(\frac{i 2 y}{\beta}\right)^{k / 2} \\
& \quad R_{k}^{(G \beta)}\left(x_{1} \sqrt{2 i y / \beta}, \ldots, x_{k} \sqrt{2 i y / \beta}, \alpha, H_{0} \sqrt{2 i y / \beta}\right) .
\end{aligned}
$$

In those cases where the imaginary unit and the singularities can cause problems, one should rather resort to the alternative expression deriving from Eq. (45),

$$
\begin{aligned}
R_{k}^{(T \beta)} & \left(x_{1}, \ldots, x_{k}, \alpha, H_{0}\right) \\
& =\int_{0}^{\infty} d t \frac{f^{(T \beta)}(t)}{(2 t)^{k / 2}} R_{k}^{(G \beta)}\left(x_{1} / \sqrt{2 t}, \ldots, x_{k} / \sqrt{2 t}, \alpha, H_{0} / \sqrt{2 t}\right) .
\end{aligned}
$$

For $H_{0}=0$ and $\beta=1$, Eq. (47) was already obtained in Ref. [17] and a similar result is given in Ref. [15] for the ensembles deriving from non-extensive entropy. Here, this is generalized to all three symmetry classes. 
Of course, supersymmetry is not needed to derive formulae (46) or (47). For $H_{0}=0$, one can now use the closed expressions for the correlation functions $R_{k}^{(G \beta)}\left(x_{1}, \ldots, x_{k}, 1,0\right)$ from Ref. [13] and calculate the correlation functions for all ensembles TOE, TUE and TSE. For $H_{0} \neq 0$, however, supersymmetry is very helpful, because it provides formula (7). As already discussed in Section 2.2, the random matrix ensemble, i.e. the matrix $\sigma$, is to some extent decoupled from the external field $H_{0}$ due to the direct product structure. This makes it possible to obtain asymptotic results for large coupling $\alpha / D$ as in Refs. [23, 24] which can then be inserted into formulae (46) and (47).

\subsection{Unitary Symmetry Class}

In the unitary symmetry class, i.e. for $\beta=2$, we can gain additional insights by following the steps outlined in Refs. [18, 19, 20]. We work with the integral transform (45) and the supersymmetric representation of the generating function (7). We absorb the parameter $\alpha$ into the supermatrix $\sigma$, which is equivalent to multiplying the variance of the Gaussian probability density with $\alpha^{2}$. The supermatrix $\sigma$ is now shifted by $x+J$ to remove these latter matrices from the superdeterminant. We then diagonalize $\sigma=u s u^{-1}$ and do the angular integration over the unitary supermatrix $u$. Here, $s=\operatorname{diag}\left(s_{11}, i s_{12}, \ldots, s_{k 1}, i s_{k 2}\right)$ is a diagonal matrix containing the eigenvalues $s_{p 1}, p=1, \ldots, k$ in the bosonic and $i s_{p 2}, p=1, \ldots, k$ in the fermionic sector. This yields [19, 20]

$$
\begin{aligned}
Z_{k}^{(G 2)}(x+J, t)=1- & \eta(x+J) \\
+ & \frac{1}{B_{k}(x+J)} \int d[s] B_{k}(s) \\
& \frac{1}{\left(2 \pi t \alpha^{2}\right)^{k}} \exp \left(-\frac{1}{2 t \alpha^{2}} \operatorname{trg}(s-x-J)^{2}\right) \\
& \operatorname{detg}^{-1}\left(s^{ \pm} \otimes 1_{N}-1_{2 k} \otimes H_{0}\right),
\end{aligned}
$$

where the function

$$
B_{k}(s)=\operatorname{det}\left[\frac{1}{s_{p 1}-i s_{q 2}}\right]_{p, q=1, \ldots, k}
$$

is the square root of the Jacobian or Berezinian arising when changing the integration variables in superspace to eigenvalue-angle coordinates. The eigenvalues in the supermatrix carry a small imaginary increment to ensure convergence, we write $s^{ \pm}$. The function $\eta(x+J)$ in Eq. (48) takes care of some Efetov-Wegner-Parisi-Sourlas contributions which are not needed here as we are mainly interested in the correlations of the imaginary parts.

Inserting Eq. (48) into the integral transform (45) and using the normalization (26), we arrive at

$$
\begin{aligned}
Z_{k}^{(T 2)}(x+J)=1 & -\eta(x+J) \\
& +\frac{1}{B_{k}(x+J)} \int d[s] B_{k}(s)
\end{aligned}
$$




$$
\begin{aligned}
& \frac{1}{\alpha^{2 k}} Q_{E}^{(T 2)}\left(\frac{1}{\alpha^{2}} \operatorname{trg}(s-x-J)^{2}\right) \\
& \quad \operatorname{detg}^{-1}\left(s^{ \pm} \otimes 1_{N}-1_{2 k} \otimes H_{0}\right),
\end{aligned}
$$

where we introduced the probability density

$$
Q_{E}^{(T 2)}(w)=\int_{0}^{\infty} f^{(T 2)}(t) \frac{1}{(2 \pi t)^{k}} \exp \left(-\frac{w}{2 t}\right) d t .
$$

Both of the expressions (28) and (51) yield probability densities in superspace, the former in the full, the latter in the eigenvalue space. This is the reason why Eq. (51) contains the power $t^{k}$ in the denominator, it arose from the integration over the unitary supermatrix. Nevertheless, we can apply the same line of arguing as in Section 3.3 and derive from Eqs. (25) and (51) the transformation formula

$$
Q_{E}^{(T 2)}(w)=\frac{2^{N / 2} \pi^{\left(N^{2}-2 k\right) / 2}}{\Gamma\left(\left(N^{2}-2 k\right) / 2\right)} \int_{0}^{\infty} P^{(T 2)}(u+w) u^{\left(N^{2}-2 k\right) / 2-1} d u,
$$

with $\mu=N^{2}$ being the number of degrees of freedom in the unitary case $\beta=2$. A comparison with the transformation formula (22) shows that the probability densities in the eigenvalue superspace follows from the one in full superspace by simply replacing the number of degrees of freedom $N^{2}$ with the reduced number $N^{2}-2 k$ where $2 k$ is the number of degrees of freedom in the eigenvalue superspace,

$$
Q_{E}^{(T 2)}(w)=\left.\frac{2^{\left(N^{2}-2 k\right) / 2}}{c^{(2)}} Q^{(T 2)}(w)\right|_{\mu=N^{2}-2 k} .
$$

One also obtains the inversion of Eq. (52) by modifying the transformation formula (23) accordingly.

Hence, we now have an exact expression (501) of the generating function for all TUE as a $2 k$ dimensional integral with a probability density given by Eqs. (52) or (53). Before going over to the correlation functions, a caveat of the same kind as discussed in Ref. [20] is in order. The limit $\alpha \rightarrow 0$ can be taken without problems in Eq. (50), thereafter the derivatives with respect to the source variables $J$ can be taken and yield the correlation functions for $\alpha=0$. Because of some interference with the EfetovWegner-Parisi-Sourlas term, this should not be done in reversed order for the case $\alpha=0$. Thus, the following discussion applies to $\alpha>0$, where the derivatives of the generating function (50) can be taken first. If we may assume that the probability density (52) does not contain inverses of source variables, we find as in Refs. [18, 19, 20]

$$
\begin{gathered}
R_{k}^{(T 2)}\left(x_{1}, \ldots, x_{k}, \alpha, H_{0}\right)=\frac{(-1)^{k}}{\pi^{k}} \int d[s] B_{k}(s) \frac{1}{\alpha^{2 k}} Q_{E}^{(T 2)}\left(\frac{1}{\alpha^{2}} \operatorname{trg}(s-x)^{2}\right) \\
\Im \prod_{p=1}^{k} \prod_{n=1}^{N} \frac{i s_{p 2}-H_{0 n}}{s_{p 1}^{ \pm}-H_{0 n}}
\end{gathered}
$$

where $H_{0 n}$ are the entries of the diagonal matrix $H_{0}$. The symbol $\Im$ denotes the proper restriction to the imaginary parts which will be explained below. As already observed in Ref. [18, the determinantal structure of the GUE correlation functions 
arises in this supersymmetry approach as an immediate consequence of the determinant structure (49). Thus, it follows form the Jacobian in superspace. The only term in the integrand which can destroy this feature for the TUE is the probability density $Q_{E}^{(T 2)}(w)$. To circumvent this problem, we use formula (51) which makes possible to advantage of explicit results for the GUE correlation functions in the presence of an external field. The formulae given in Ref. [19, 20] are slightly more general than what we need in the present context, because they also contains an integral over the probability density $P_{0}\left(H_{0}\right)$ of the external field $H_{0}$. However, a $\delta$ function $P_{0}\left(H_{0}\right)$ trivially yields

$$
R_{k}^{(G 2)}\left(x_{1}, \ldots, x_{k}, t \alpha^{2}, H_{0}\right)=\operatorname{det}\left[C_{N}\left(x_{p}, x_{q}, t \alpha^{2}, H_{0}\right)\right]_{p, q=1, \ldots, k} .
$$

The kernel is given as the double integral

$$
\begin{gathered}
C_{N}\left(x_{p}, x_{q}, t \alpha^{2}, H_{0}\right) \\
=-\frac{1}{2 \pi^{2} t \alpha^{2}} \int_{-\infty}^{+\infty} \int_{-\infty}^{+\infty} \frac{d s_{1} d s_{2}}{s_{1}-i s_{2}} \exp \left(\frac{\left(i s_{2}-x_{q}\right)^{2}}{2 t \alpha^{2}}-\frac{\left(s_{1}-x_{p}\right)^{2}}{2 t \alpha^{2}}\right) \\
\Im \prod_{n=1}^{N} \frac{i s_{2}-H_{0 n}}{s_{1}^{-}-H_{0 n}},
\end{gathered}
$$

where we drop the indices $p$ and $q$ of the integration variables. The present notation slightly deviates, in a hopefully self-explanatory way, from the previous one. We now have the variance $t \alpha^{2}$ as an argument, because it contains the parameter $\alpha$ after the above mentioned changes of integration variables. The process of going over to the imaginary parts of the correlation functions amounts to inserting the definition

$$
\Im \prod_{n=1}^{N} \frac{i s_{2}-H_{0 n}}{s_{1}^{-}-H_{0 n}}=\frac{1}{i 2}\left(\prod_{n=1}^{N} \frac{i s_{2}-H_{0 n}}{s_{1}^{-}-H_{0 n}}-\prod_{n=1}^{N} \frac{i s_{2}-H_{0 n}}{s_{1}^{+}-H_{0 n}}\right) .
$$

We notice that the term $1 /\left(s_{1}-i s_{2}\right)$ in the integrand of Eq. (566) is the remainder of the Jacobian. After Refs. [18, 19, 20], such double integral expressions were also derived by other authors.

We combine these findings and arrive at

$$
\begin{aligned}
R_{k}^{(T 2)} & \left(x_{1}, \ldots, x_{k}, \alpha, H_{0}\right) \\
& =\int_{0}^{\infty} d t \frac{f^{(T 2)}(t)}{(2 \pi t)^{k}} \operatorname{det}\left[C_{N}\left(x_{p}, x_{q}, t \alpha^{2}, H_{0}\right)\right]_{p, q=1, \ldots, k}
\end{aligned}
$$

which is an exact representation of the TUE correlation functions for finite level number in the presence of an external field. For convenience, we derived this result using the spread function $f^{(T 2)}(t)$. However, depending on the specific form of the probability density $Q_{E}^{(T 2)}(w)$ given in Eqs. (52) and (153), one might want to prefer another integral representation. Any such representation which involves a Gaussian will lead to a result of the form (58), but with the spread function replaced by another function. It should also be mentioned that the result (58) can alternatively be derived starting directly from Eq. (47). This, however, would not lead to the probability density $Q_{E}^{(T 2)}(w)$ and the corresponding observation concerning the reduced number of degrees of freedom. 
Furthermore, we notice that the double integral (56) for the kernel can be evaluated in closed form. For the sake of completeness, we give this result in Appendix B.

\section{Summary and Conclusions}

We showed that the all norm-dependent random matrix ensembles TOE, TUE and TSE have a supersymmetric representation. Hence, supersymmetry is by no means restricted to Gaussian probability densities. We mapped the functions generating the $k$ point correlation functions onto their supersymmetric analogues. All our results include an external field. No approximation was made, all expressions are exact and for finite level number. We derived transformation formulae which yield the probability density in superspace as one-dimensional integral involving the probability density in ordinary space. These formulae state the most important conceptual insight of this contribution. We emphasize that only the existence, but not the explicit knowledge of the Fourier integral or the spread function is needed to obtain the probability density in superspace. The transformation formulae clarify the mechanism of how supersymmetry manages to reduce the number of degrees of freedom. We worked out several examples. Remarkably, the functional forms of the probability densities tends to be very similar in ordinary and superspace. In particular, this is so for the whole family of ensembles deriving from a non-extensive entropy principle.

From a practical viewpoint, our most important findings are a series of exact results for the correlation functions which can be used in applications. Different limits for the level number or other parameters can now be studied depending on the ensemble of interest. The most explicit formulae are for the TUE where we employed the determinant structure of the GUE correlations. We also derived a probability density for the TUE in the superspace of eigenvalues.

Can supersymmetry be applied to ensembles which are more general than the norm-dependent ones? - Indeed, a supersymmetric representation is possible under quite general conditions. However, as this construction requires a completely different approach, we defer it to another contribution [34.

\section{Acknowledgments}

I thank Frieder Kalisch and Heiner Kohler for fruitful discussions. I acknowledge financial support from Det Svenska Vetenskapsrådet.

\section{Appendix A. Moments of Norm-dependent Probability Densities}

The following calculation, although rather straightforward, yields an interesting side result which might be useful in other applications. This is why we sketch the calculation here. Inserting the diagonalizations $H=U x U^{-1}$ with $x=\operatorname{diag}\left(x_{1}, \ldots, x_{N}\right)$ and a 
double degeneracy for $\beta=4$, we find from the definition of the moments in Eqs. (12)

$$
\begin{aligned}
M_{\nu}^{(T \beta)}=\pi^{\beta N(N-1) / 4} & \frac{\Gamma^{N}(1+\beta / 2)}{\prod_{n=1}^{N} \Gamma(1+n \beta / 2)} \\
& \int P^{(T \beta)}\left(\operatorname{Tr} x^{2}\right)\left(\operatorname{Tr} x^{2}\right)^{\nu}\left|\Delta_{N}(x)\right|^{\beta} d[x],
\end{aligned}
$$

where $\Delta_{N}(x)=\prod_{n<m}\left(x_{n}-x_{m}\right)$ is the Vandermonde determinant. The constant in front of the eigenvalue integral contains the result of the integration over $U$ and also some factors stemming from the Jacobian of the transformation to eigenvalue-angle coordinates. We view the eigenvalues as components of a vector $\vec{r}=\left(x_{1}, \ldots, x_{N}\right)$ in $N$ dimensions and introduce polar coordinates $\vec{r}=r \vec{e}$ where $r$ is the length and $\vec{e}$ a unit vector depending on $N-1$ angles. The volume element reads $d[x]=d^{N} x=r^{N-1} d r d \Omega$ where $d \Omega$ is the infinitesimal solid angle. Because of $r^{2}=\operatorname{Tr} x^{2}$, we have

$$
\begin{aligned}
M_{\nu}^{(T \beta)}=\pi^{\beta N(N-1) / 4} & \frac{\Gamma^{N}(1+\beta / 2)}{\prod_{n=1}^{N} \Gamma(1+n \beta / 2)} \\
& \int\left|\Delta_{N}(\vec{e})\right|^{\beta} d \Omega \int_{0}^{\infty} r^{\nu+N-1+\beta N(N-1) / 2} P^{(T \beta)}\left(r^{2}\right) d r .
\end{aligned}
$$

The angular integral can be infered by inserting the Gaussian probability density (4)

and considering $\nu=0$, i.e. $M_{0}^{(T \beta)}=1$. The radial integral can then be done and we find as an interesting side result

$$
\int\left|\Delta_{N}(\vec{e})\right|^{\beta} d \Omega=\frac{\pi^{N / 2} \prod_{n=1}^{N} \Gamma(1+n \beta / 2)}{2^{\beta N(N-1) / 4-1} \Gamma^{N}(1+\beta / 2) \Gamma(\mu / 2)} .
$$

Putting $u=r^{2}$ and collecting everything we arrive at the second of Eqs. (12).

\section{Appendix B. Evaluation of the Kernel}

We start by observing that the determinant can be written in the form

$$
\begin{aligned}
\prod_{n=1}^{N} \frac{i s_{2}-H_{0 n}}{s_{1}^{-}-H_{0 n}} & =\prod_{n=1}^{N}\left(1+\frac{i s_{2}-s_{1}}{s_{1}^{-}-H_{0 n}}\right) \\
& =1+\sum_{n=1}^{N} \frac{i s_{2}-s_{1}}{s_{1}^{-}-H_{0 n}} \sum_{m=0}^{N-1} \frac{\left(i s_{2}-s_{1}\right)^{m}}{\left.\prod_{m^{\prime} \in \Omega_{n, m}^{(N)}}^{\left(H_{0 n}\right.}-H_{0 m^{\prime}}\right)} .
\end{aligned}
$$

Here, $\Omega_{n, m}^{(N)}$ is a subset of the $N-1$ indices remaining when removing the index $n$ from the original $N$ indices such that $m$ pairs $\left(n, m^{\prime}\right)$ are formed. This can also be formulated in terms of symmetric functions. For example, in the case $N=3$ and $n=1$, one has

$$
\begin{aligned}
& \sum_{m=0}^{2} \frac{\left(i s_{2}-s_{1}\right)^{m}}{\prod_{m^{\prime} \in \Omega_{1, m}^{(3)}}\left(H_{0 n}-H_{0 m^{\prime}}\right)} \\
& \quad=1+\frac{i s_{2}-s_{1}}{H_{01}-H_{02}}+\frac{i s_{2}-s_{1}}{H_{01}-H_{03}}+\frac{\left(i s_{2}-s_{1}\right)^{2}}{\left(H_{01}-H_{02}\right)\left(H_{01}-H_{03}\right)} .
\end{aligned}
$$


This yields a most convenient expression containing only $\delta$ functions,

$$
\begin{aligned}
& \Im \prod_{n=1}^{N} \frac{i s_{2}-H_{0 n}}{s_{1}^{-}-H_{0 n}}=\pi \sum_{n=1}^{N}\left(i s_{2}-s_{1}\right) \delta\left(s_{1}-H_{0 n}\right) \\
& \sum_{m=0}^{N-1} \frac{\left(i s_{2}-s_{1}\right)^{m}}{\prod_{m^{\prime} \in \Omega_{n, m}^{(N)}}^{(N)}\left(H_{0 n}-H_{0 m^{\prime}}\right)},
\end{aligned}
$$

which facilitates the evaluation of the $s_{1}$ integral in Eq. (56). Importantly, the difference $s_{1}-i s_{2}$ also disappears in the denominator and the remaining $s_{2}$ integration simply becomes

$$
\begin{aligned}
\int_{-\infty}^{+\infty}\left(i s_{2}-H_{0 n}\right)^{m} \exp \left(\frac{\left(i s_{2}-x_{q}\right)^{2}}{2 t \alpha^{2}}\right) d s_{2} & \\
= & \frac{{\sqrt{2 t \alpha^{2}}}^{m+1} \sqrt{\pi}}{2^{m}} \mathbf{H}_{m}\left(\frac{x_{q}-H_{0 n}}{\sqrt{2 t \alpha^{2}}}\right),
\end{aligned}
$$

with $\mathbf{H}_{m}(z)$ denoting the Hermite polynomial of degree $m$. Collecting everything, we find

$$
\begin{aligned}
C_{N}\left(x_{p}, x_{q}, t \alpha^{2}, H_{0}\right)=\sum_{m=0}^{N-1}\left(\frac{t \alpha^{2}}{2}\right)^{m / 2} \sum_{n=1}^{N} \frac{\exp \left(\left(H_{0 n}-x_{p}\right)^{2} / 2 t \alpha^{2}\right)}{\sqrt{2 \pi t \alpha^{2}}} \\
\frac{\mathbf{H}_{m}\left(\left(x_{q}-H_{0 n}\right) / \sqrt{2 t \alpha^{2}}\right)}{\prod_{m^{\prime} \in \Omega_{n, m}^{(N)}}\left(H_{0 n}-H_{0 m^{\prime}}\right)}
\end{aligned}
$$

We notice that the first sum extends from zero to $N-1$, exactly as in the case of the GUE without external field.

\section{References}

[1] K.B. Efetov, Adv. Phys. 32 (1983) 53.

[2] K.B. Efetov K, Supersymmetry in Disorder and Chaos, Cambridge University Press, Cambridge (1997).

[3] J.J.M. Verbaarschot, H.A. Weidenmüller and M. Zirnbauer, Phys. Rep. 129 (1985) 367.

[4] T. Guhr, A. Müller-Groeling and H.A. Weidenmüller, Phys. Rep. 299 (1998) 189.

[5] F. Haake, Quantum Signatures of Chaos, 2nd edition, (Springer Verlag, Berlin 2001).

[6] T. Guhr, Random Matrix Theory in Physics, Encyclopedia of Mathematical Physics, eds. J.-P. Franoise, G.L. Naber and Tsou S.T, (Elsevier, Oxford 2006), vol. 4, pp. 338.

[7] M. Zirnbauer, Supersymmetry Methods in Random Matrix Theory, Encyclopedia of Mathematical Physics, eds. J.-P. Franoise, G.L. Naber and Tsou S.T, (Elsevier, Oxford 2006), vol. 5, pp. 151, math-ph/0404057

[8] E. Brézin and A. Zee, Nucl. Phys. B402 (1993) 613.

[9] E. Brézin and A. Zee, C. R. Acad. Sci. 17 (1993) 735.

[10] J. Ambjørn, J. Jurkiewicz and Yu.M. Makeenko, Phys. Lett. B251 (1990) 517.

[11] E. Brézin, C. Itzykson, G. Parisi and J. Zuber, Commun. Math. Phys. 59 (1978) 35.

[12] L. Laloux, P. Cizeau, J.P. Bouchaud and M. Potters, Phys. Rev. Lett. 83 (1999) 1467.

[13] M.L. Mehta, Random Matrices, 3rd edition, (Academic Press, New York 2004).

[14] F. Toscano, R.O. Vallejos and C. Tsallis, Phys. Rev. E69 (2004) 066131.

[15] A.C. Bertuola, O. Bohigas and M.P. Pato, Phys. Rev. E70 (2004) 065102(R).

[16] G. Hackenbroich and H.A. Weidenmüller, Phys. Rev. Lett. 74 (1995) 4418. 
[17] K.A. Muttalib and J.R. Klauder, Phys. Rev. E71 (2005) 055101(R).

[18] T. Guhr, J. Math. Phys. 32 (1991) 336.

[19] T. Guhr, Phys. Rev. Lett. 76 (1996) 2258.

[20] T. Guhr, Ann. Phys. (NY) 250 (1996) 145, cond-mat/9510052

[21] T. Guhr and H. Kohler, math-ph/0012047

[22] T. Guhr and H. Kohler, J. Math. Phys 43 (2002) 2741

[23] T. Guhr and H.A. Weidenmüller, Ann. Phys. (NY) 193 (1989) 472.

[24] K. Frahm, T. Guhr and A. Müller-Groeling, Ann. Phys. (NY) 270 (1998) 292.

[25] G. Parisi and G. Sourlas, Phys. Rev. Lett. 43 (1979) 744.

[26] F. Wegner, Z. Phys. B49 (1983) 297.

[27] F. Constantinescu and H.F. de Groote, J. Math. Phys. 30 (1989) 981.

[28] M.J. Rothstein, Trans. Am. Math. Soc. 299 (1987) 387.

[29] F.J. Dyson, J. Math. Phys 1 (1962) 140.

[30] F.J. Dyson, J. Math. Phys 1 (1962) 1191.

[31] G. Le Caër and R. Delannay, Phys. Rev. E59 (1999) 6281.

[32] G. Akemann, G.M. Cicuta, L. Molinari and G. Vernizzi, Phys. Rev. E59 (1999) 1489.

[33] I.S. Gradshteyn and I.M. Ryzhik, Table of Integrals, Series and Products, (Academic Press, New York, 2000).

[34] T. Guhr, Arbitrary Rotation Invariant Random Matrix Ensembles and Supersymmetry, submitted for publication. 